\title{
Talento de los estudiantes de ingeniería para emprender actividades de negocio
}

Talent of the students of engineering to undertake activities of business

\author{
Alfredo Edgar López Jiménez¹, Ulises Robert Martínez Chafalote', José Luis Pérez Ramírez', Aldo Manuel \\ Canales Changanaqui', Luis Maurilio Espinoza Flores', Lino Rolando Rodríguez Alegre', William Fredy López \\ Jiménez', Elver Stalin Ticliahuanca Calderón', Deysi America Delgado Campos ${ }^{1}$
}

\section{RESUMEN}

Objetivo: Analizar el Talento Cognitivo de los Estudiantes de Ingeniería y su relación con la Experiencia en Algún Negocio. Metodos: La investigación fue de tipo Aplicada, de nivel correlacional, de enfoque cuantitativo, porque se aplico el método deductivo e inductivo. Se utilizó el modelo del diseño de investigación descriptivo correlacional. La población del estudio fue de 1422 estudiantes de la Facultad de Ingeniería Industrial, Sistemas e Informática, del que se calculó un tamaño de muestra final de 250 estudiantes. Resultados: A nivel descriptivo sobre la primera variable denominada el Talento Cognitivo de los estudiantes, se consideró los factores como: Conocimientos en Economía, Conocimientos en Negocios y Conocimientos en Gestión; los resultados indican que tienen niveles Básicos de Conocimientos, representando a la mayoría con un porcentaje del $58,8 \%$. Sobre la segunda variable denominada Experiencia en Algún Negocio, los resultados muestran que son las Ventas las experiencias más comunes representados con un porcentaje del $56,0 \%$. A nivel inferencial, las pruebas de hipótesis para cada factor arrojaron una significación de muestra de 0,000, menor al 0,05 probabilistico, lo que confirma los planteamientos iniciales. Conclusiones: A la luz de los resultados se comprueba que existen factores cognitivos que predisponen a los estudiantes de ingeniería para emprender actividades de negocios. Estos resultados nos revela la oportunidad que debe de aprovecharse para fortalecer los conocimientos de los estudiantes de ingeniería en el mundo de los negocio a nivel formal, de tal manera que se contribuya en nuestra comunidad con el desarrollo de la economía local y nacional.

Palabras clave: Talento, conocimientos, economía, negocio, gestión, ventas, servicios, producción.

\section{ABSTRACT}

Objective: To evaluate the cognitive talent of students of engineering and its relationship with some business experience. Methods: Deductive and inductive method was applied. The model of the descriptive research design was used correlational. The study population was 1422 students from the Faculty of Industrial Engineering, systems and computer science, from which a final sample of 250 students size was calculated. Results: Descriptive level on the first so-called variable cognitive talent of the students, was considered the factors as: knowledge economy, knowledge in business and management; the results indicate that they have knowledge of basic levels, representing most with $58.8 \%$. On the second variable named experience in any business, the results show that sales are the most common experiences represented a percentage of $56.0 \%$. Inferential level, tests of hypotheses for each factor threw a significance of 0.000 , less than the 0.05 probability sample, thus confirming the initial approaches. Conclusions: In the light of the results is checked that there are cognitive factors that predispose to engineering students to undertake business activities. These results reveal the opportunity which musttake to streng then the knowledge of students of engineering in the world of business them at a formal level, in such a way that it contributes in our community with the development of the local economy and National.

Keywords: Talent, knowledge, economy, business, management, sales, services, production.

${ }^{1}$ Universidad Nacional José Faustino Sánchez Carrión. Huacho, Perú. 


\section{INTRODUCCIÓN}

En nuestro país el desempleo es un problema social vigente, esta situación afecta en especial a los jóvenes, sobre todo en edad universitaria, quienes disponen de mucha energía y mucha ilusión, pero por lo general hay pocas oportunidades para emplearse en alguna empresa ó algún negocio.

El diario Perú21 (2015) describe los resultados de una investigación realizada por la empresa Aptitus.com; el $20 \%$ de peruanos entre 18 y 24 años tienen trabajo, esta situación obedece a las pocas oportunidades laborales que ofrece el mercado $(58 \%)$, así como a su falta de experiencia (46\%), lo que probablemente los convierta en menos atractivos para las empresas. Aunque un pequeño grupo $(9 \%)$ reconoció que esto se debe a su falta de iniciativa propia. De este grupo de jóvenes el $64 \%$ manifestó no sentirse a gusto con su sueldo, esta realidad nos indica que los jóvenes están expuestos a ser atraídos por personas que actúan al margen de la ley con la ilusión de ganar dinero de manera rápida.

La inserción laboral para los jóvenes que acaban de terminar de estudiar una carrera técnica o universitaria puede tornarse difícil. Esto se debe a la necesidad de las empresas por contar, con personal con mayor experiencia o a que estas han tomado la iniciativa de formar a sus futuros ejecutivos desde la etapa de las prácticas pre profesionales.

Pero, ¿qué ocurre con ese porcentaje de jóvenes que ya tiene un empleo?, el estudio de Aptitus.com reveló datos interesantes con respecto a este grupo. Por ejemplo, el $88 \%$ de estos jóvenes señalaron haber conseguido un puesto de trabajo en menos de un año tras egresar de su universidad o instituto. En tanto que el $9 \%$ demoró entre 1 y 3 años, y el restante, más de 3 años. Finalmente, el $75 \%$ manifestó sentirse a gusto con su empleo.

Ante esta situación es necesario saber si los jóvenes están preparados o tienen alguna capacidad para generar su propia fuente de trabajo, para iniciar alguna actividad económica y sostenerse en el tiempo.

El desempleo es definido como la ausencia del empleo u ocupación; están desocupadas o desempleadas aquellas personas que no encuentran quién las contraten como trabajadores (Garavito, 1989). Para que exista una situación de ¿desempleo? es necesario que la persona no sólo desee trabajar sino que, además, acepte los salarios actuales que se están pagando en un momento dado.

Los economistas han descrito las causas del desempleo como friccionales, temporales, estructurales y cíclicas. El desempleo friccional, se produce porque los trabajadores que están buscando un empleo no la encuentran de inmediato, mientras que están buscando trabajo son contabilizados como desempleados. En cambio, el desempleo temporal, se produce cuando las industrias tienen una temporada baja, como durante el invierno. Por otro lado, el desempleo estructural, se debe a un desequilibrio entre el tipo de trabajadores que requieren los empresarios y el tipo de trabajadores que buscan trabajo. Este desequilibrio pueden deberse a que la capacitación y característica de los personales no son adecuadas. Por último, el desempleo cíclico, es el resultado de una falta de demanda general de trabajo. Cuando el ciclo económico cae, la demanda de bienes y servicios cae también y, por lo tanto, se despide a los trabajadores.

El trabajo les brinda a las personas la posibilidad de lograr ingresos, por tanto acceder a bienes y servicios, y así satisfacer sus necesidades. En la medida que logren satisfacer mejor sus necesidades, mejor será su nivel de vida. Es por eso que existe un fuerte vínculo entre el desempleo y la pobreza. Ahora bien, no sólo es importante el tener empleo, sino la calidad del empleo, es decir, el salario, la jornada laboral, las condiciones de empleo, la estabilidad, la seguridad social, entre muchos otros elementos.

Por otra parte para que un joven se convierta en un emprendedor de Negocios, es necesario que disponga de ciertos Conocimientos y Habilidades, se le denomina el Talento Cognitivo del joven. El Talento se refiere a la Inteligencia ó capacidad de entender que tiene el joven, también se refiere a la Aptitud ó capacidad que tiene el joven para el desempeño de una ocupación

Sobre este punto, Espitía y Barragán (2004), sostiene que para competir dentro de un entorno globalizado, altamente competitivo, de 
transformaciones profundas, aceleradas y dinámicas, se exige un cambio radical en las creencias, costumbres y valores de la empresa, donde las personas deben asumir roles diferentes y adoptar una visión de mayor apertura y flexibilidad ante el cambio. Para lograr esto se debe luchar por obtener el compromiso del talento humano el cual solo se alcanzará si existe equilibrio y justicia empresarial. El verdadero tesoro que puede generar sostenibilidad y ventaja competitiva a la empresa es el talento humano.

Por esta afirmación en importante conocer la capacidad del Talento que disponen los jóvenes universitarios para emprender actividades de negocios, con este motivo se desarrollo la presente investigación.

La hipótesis de la investigación.

El Talento cognitivo se relaciona con la Experiencia de los estudiantes de ingeniería en algún negocio.

Siendo el objetivo analizar la relación entre el Talento Cognitivo con la Experiencia en Algún Negocio de los estudiantes de ingeniería.

\section{MATERIAL Y MÉTODOS}

Nuestra investigación se desarrolló en los ambientes de la Universidad Nacional José Faustino Sánchez Carrión, la recolección de datos se realizó en los ambientes de las aulas de la Facultad de Ingeniería Industrial, Sistemas e Informática perteneciente a la Universidad Nacional José Faustino Sánchez Carrión, con participación de los estudiantes de esta Facultad. Ellos constituyeron nuestra población cuya característica fue finita y que según los registros oficiales son 1422 estudiantes matriculados en forma oficial en sus 4 Escuelas Profesionales; Ingeniería Industrial, Ingeniería de Sistemas, Ingeniería Informática e Ingeniería Electrónica, de la Facultad en mención.

El tamaño de muestra calculado fue de 250 estudiantes matriculados, se utilizó el Muestreo Simple ó Aleatorio, el cual cumplió con la característica de ser probabilístico.

Al respecto Sánchez y Reyes (1998), sostienen que en este caso todos los miembros de una población tienen iguales posibilidades de ser elegidos para conformar una muestra. Estos estudiantes fueron encuestadas al azar en el interior de las aulas de la Facultad en estudio. Por otra parte $\mathrm{Nel}$ (2010), manifiesta que cuando todos los elementos de una población tienen la misma probabilidad de ser elegidos, los individuos que formarán parte de una muestra se elegirán al azar.

El Instrumento utilizado ha sido un modelo de encuesta elaborado por los propios autores, con cuestionario refirido a factores para medir el Talento Cognitivo de los Estudiantes, tales como; Conocimientos en Economía, Conocimientos en Negocio, Conocimientos en Gestión y Experiencia Alguna en Negocios. Este instrumento fue evaluado con el alpha de cronbach, cuyo coeficiente resulto con un valor del 0,712 , el cual lo ubica en una categoría de alta confiabilidad, así mismo tiene una validación de juicio de expertos del $92,6 \%$, que lo valida como muy bueno.

Los métodos empleados fueron el deductivo e inductivo, el primero porque hace un análisis de la realidad problemática a nivel general y se llega a un análisis específico en este caso el Talento Cognitivo de los Estudiantes para emprender actividades de negocios y con lo cual se aplica técnicas de la estadística descriptiva para analizar los datos cualitativos recolectados, a nivel de frecuencias absolutas y porcentuales; el segundo método se evidencia con las pruebas de hipótesis estadístico que analizan a la muestra y se infiere la realidad a nivel general; y con aplicación de las técnicas de la estadística inferencial para analizar los datos recolectados, como es la Prueba de Hipótesis de Independencia, a un nivel de significación del $5 \%$, el cual nos permite tomar decisiones concretas sobre las hipótesis formuladas en la investigación.

\section{RESULTADOS}

En primer lugar, se presentan los resultados descriptivos de la investigación.

En relación al Nivel de Conocimientos en Economía de los estudiantes de ingeniería, se considero a tres categorías como medidas para este indicador, Avanzado, Intermedio y Básico

En la Tabla 1 y Figura 1, se muestran los resultados sobre el Nivel de Conocimientos en 
Economía de los estudiantes de ingeniería; en ella se visualiza que en primer lugar están los estudiantes cuyo conocimiento es Básico, ellos representan a la mayoría con un porcentaje del $56,4 \%$, en segundo lugar están los que pertenecen a la categoría Intermedia, ellos están representados con un $39,6 \%$. Estos resultados nos expresan que los estudiantes de ingeniería tienen conocimientos bajos sobre las funciones que establece la economía en general.

Tabla 1. Nivel de conocimientos en economía de los estudiantes de ingeniería

\begin{tabular}{lcc}
\hline \multirow{2}{*}{$\begin{array}{c}\text { Conocimientos } \\
\text { en Economía }\end{array}$} & \multicolumn{2}{c}{ Frecuencia } \\
\cline { 2 - 3 } & Alumnos & Porcentaje \\
\hline Avanzado & 10 & $4,0 \%$ \\
Intermedio & 99 & $39,6 \%$ \\
Básico & 141 & $56,4 \%$ \\
\hline \multicolumn{1}{c}{ Total } & 250 & $100,0 \%$ \\
\hline
\end{tabular}

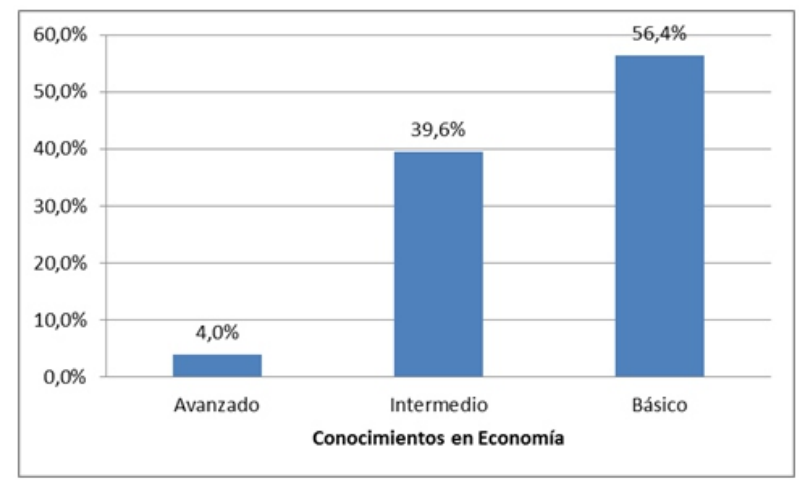

Figura 1. Nivel de Conocimientos en Economía de los estudiantes de ingeniería

En relación al Nivel de Conocimientos en Negocios de los estudiantes de ingeniería, se considero a tres categorías como medidas para este indicador, Avanzado, Intermedio y Básico

En la Tabla 2 y Figura 2, se muestran los resultados sobre el Nivel de Conocimientos en Negocios de los estudiantes de ingeniería; en ella se aprecia que en primer lugar están los estudiantes cuyo conocimiento es Básico, ellos representan a la mayoría con un porcentaje del $56,0 \%$, en segundo lugar están los que pertenecen a la categoría Intermedia, ellos están representados con un $41,2 \%$. Estos resultados nos expresan que los estudiantes de ingeniería tienen conocimientos limitados sobre las actividades que implican el mundo de los negocios en forma general.

Tabla 2. Nivel de Conocimientos en Negocios de los estudiantes de ingeniería

\begin{tabular}{lcc}
\hline \multicolumn{1}{c}{$\begin{array}{c}\text { Conocimientos } \\
\text { en Negocios }\end{array}$} & Alumnos & Porcentaje \\
\hline Avanzado & 7 & $2,8 \%$ \\
Intermedio & 103 & $41,2 \%$ \\
Básico & 140 & $56,0 \%$ \\
\hline \multicolumn{1}{c}{ Total } & 250 & $100,0 \%$ \\
\hline
\end{tabular}

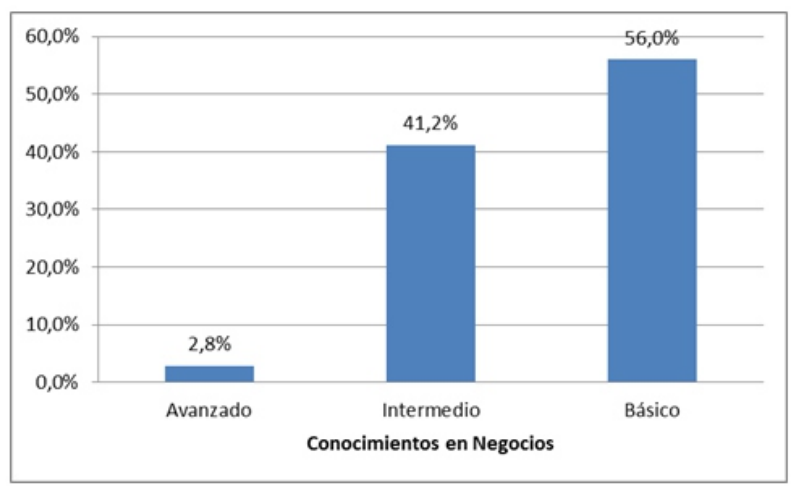

Figura 2. Nivel de Conocimientos en Negocios de los estudiantes de ingeniería

En relación al Nivel de Conocimientos en Gestión de los estudiantes de ingeniería, se considero a tres categorías como medidas para este indicador, Avanzado, Intermedio y Básico

En la Tabla 3 y Figura 3, se muestran los resultados sobre el Nivel de Conocimientos en Gestión de los estudiantes de ingeniería; en ella se observa que en primer lugar están los estudiantes cuyo conocimiento es Básico, ellos representan a la mayoría con un porcentaje del $63,2 \%$, en segundo lugar están los que pertenecen a la categoría Intermedia, ellos están representados con un 32,8\%. Estos resultados nos expresan que los estudiantes de ingeniería tienen conocimientos restringidos sobre las acciones que involucran el mundo de la gestión en general. 
Tabla 3. Nivel de Conocimientos en Gestión de los estudiantes de ingeniería

\begin{tabular}{lcc}
\hline \multirow{2}{*}{$\begin{array}{c}\text { Conocimientos } \\
\text { en Gestión }\end{array}$} & \multicolumn{2}{c}{ Frecuencia } \\
\cline { 2 - 3 } & Alumnos & Porcentaje \\
\hline Avanzado & 10 & $4,0 \%$ \\
Intermedio & 82 & $32,8 \%$ \\
Básico & 158 & $63,2 \%$ \\
\hline \multicolumn{1}{c}{ Total } & 250 & $100,0 \%$ \\
\hline
\end{tabular}

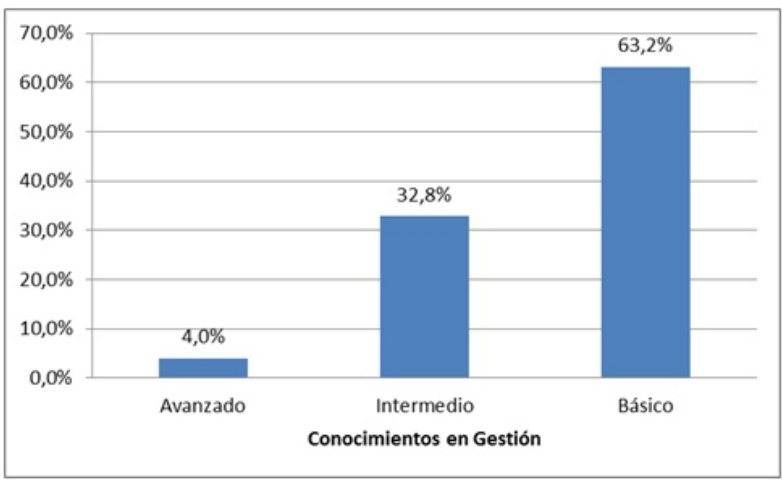

Figura 3. Nivel de Conocimientos en Gestión de los estudiantes de ingeniería

El resumen sobre la primera variable; Talento Cognitivo del Estudiante, a través de sus indicadores: Conocimientos en Economía, Conocimientos de Negocio, Conocimientos en Gestión, se muestra en tres categorías, Avanzado, Intermedio y Básico.

En la Tabla 4 y Figura 4, se muestran los resultados sobre el Talento Cognitivo de los estudiantes de ingeniería; en ella se visualiza que en primer lugar están los estudiantes cuyo talento es Básico, ellos representan a la mayoría con un porcentaje del $58,8 \%$, en segundo lugar están los que pertenecen a la categoría Intermedia, ellos están representados con un $38,4 \%$. Estos resultados nos expresan que los estudiantes de ingeniería tienen el Talento Cognitivo con limitaciones para desarrollar alguna actividad de Negocio con la formalidad que la leyes de país establece.
Tabla 4. Talento Cognitivo de los estudiantes de ingeniería

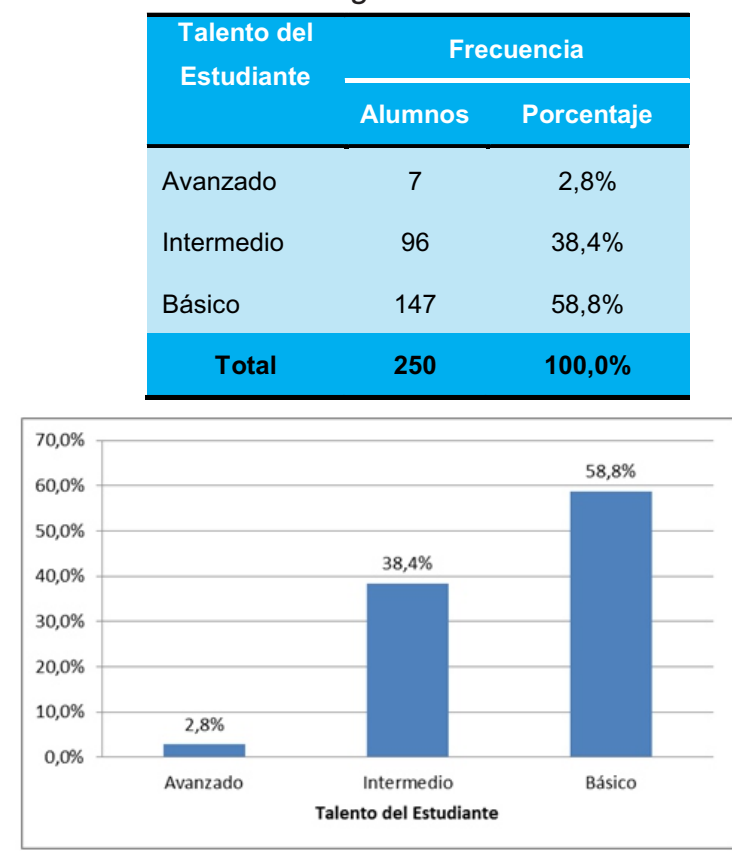

Figura 4. Talento Cognitivo de los estudiantes de ingeniería

Sobre la segunda variable; Experiencia en algún Negocio de los estudiantes de ingeniería, se utilizó tres medidas nominales, Venta, Servicio y Producción.

En la Tabla 5 y Figura 5 , se muestran los resultados sobre la Experiencia en algún Negocio de los estudiantes de ingeniería; en ella se aprecia que en primer lugar están los estudiantes cuya experiencia es en Ventas, ellos representan a la mayoría con un porcentaje del $56,0 \%$, en segundo lugar están los que tienen experiencia en Servicio, ellos están representados con un $31,2 \%$. Estos resultados nos manifiestan que la experiencia más común que tienen los estudiantes de ingeniería, es en Ventas, lo cual indica que se debe aprovechar esta fortaleza para mejorar el comercio de la ciudad

Tabla 5. Experiencia en Algún Negocio de los estudiantes de ingeniería

\begin{tabular}{lcc}
\hline Experiencia en & \multicolumn{2}{c}{ Frecuencia } \\
\cline { 2 - 3 } Algún Negocio & Alumnos & Porcentaje \\
\cline { 2 - 3 } & 140 & $56,0 \%$ \\
Venta & 78 & $31,2 \%$ \\
Servicio & 32 & $12,8 \%$ \\
Producción & 250 & $100,0 \%$ \\
\hline \multicolumn{1}{c}{ Total } &
\end{tabular}




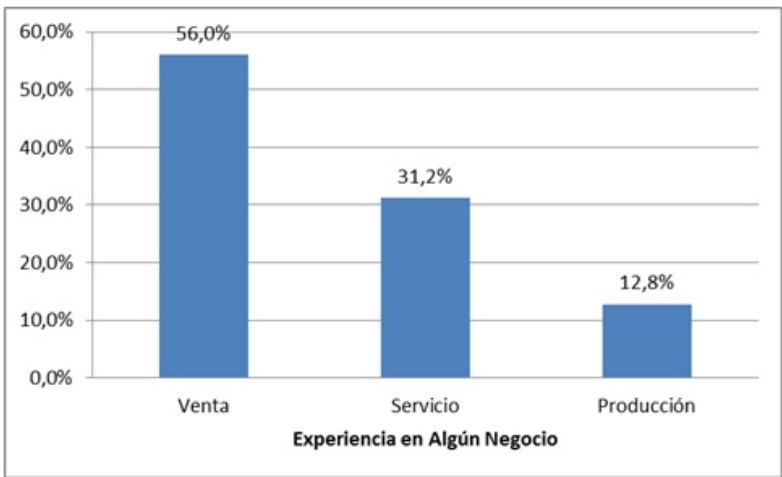

Figura 3. Experiencia en Algún Negocio de los estudiantes de ingeniería

En segundo lugar, se presentan los resultados inferenciales de la investigación.

La primera prueba de hipótesis se realizó con la tabla de contingencia, Tabla 6.

Hn: El Nivel de Conocimientos en Economía No se relaciona con la Experiencia en Algún Negocio de los estudiantes de ingeniería.

Ha: El Nivel de Conocimientos en Economía Si se relaciona con la Experiencia en Algún Negocio de los estudiantes de ingeniería.

Tabla 6. Conocimientos en economía y experiencia en algún negocio

\begin{tabular}{lcccc}
\hline \multirow{2}{*}{$\begin{array}{c}\text { Conocimientos } \\
\text { En Economía }\end{array}$} & \multicolumn{5}{c}{ Experiencia en Algún Negocio } \\
\cline { 2 - 5 } & Producción & Servicio & Venta & Total \\
\hline Básico & 10 & 36 & 95 & 141 \\
Intermedio & 18 & 38 & 43 & 99 \\
Avanzado & 4 & 4 & 2 & 10 \\
\multicolumn{1}{r}{ Total } & 32 & 78 & 140 & 250 \\
\hline \multicolumn{7}{r}{}
\end{tabular}

Tabla 7. Correlación de Rho de Spearman

\begin{tabular}{|c|c|c|c|c|}
\hline & & & $\begin{array}{c}\text { CONOCIMIENTOS DE } \\
\text { ECONOMÍA }\end{array}$ & $\begin{array}{l}\text { EXPERIENCIA } \\
\text { EN ALGÚN } \\
\text { NEGOCIO }\end{array}$ \\
\hline \multirow{3}{*}{ Rho de Spearman } & $\begin{array}{l}\text { CONOCIMIENTOS } \\
\text { DE ECONOMÍA }\end{array}$ & $\begin{array}{l}\text { Coeficiente de correlación } \\
\text { Sig. (bilateral) } \\
\text { N }\end{array}$ & $\begin{array}{l}1,000 \\
\dot{250}\end{array}$ & $\begin{array}{c}-0,290^{* *} \\
0,000 \\
250\end{array}$ \\
\hline & \multirow{2}{*}{$\begin{array}{l}\text { EXPERIENCIA EN } \\
\text { ALGÚN NEGOCIO }\end{array}$} & $\begin{array}{l}\text { Coeficiente de correlación } \\
\text { Sig. (bilateral) }\end{array}$ & $\begin{array}{c}-0,290^{* *} \\
0,000\end{array}$ & 1,000 \\
\hline & & $\mathrm{N}$ & 250 & 250 \\
\hline
\end{tabular}

**. La correlación es significativa al nivel 0,01 (bilateral).

Con una significación de la muestra de 0,000, menor al 0,01 de significación probabilística, se rechaza la hipótesis nula y en su lugar se acepta la hipótesis alternativa, es decir se demuestra que el Nivel de Conocimientos en Economía Si se relaciona con la Experiencia en Algún Negocio de los estudiantes de ingeniería.

La segunda prueba de hipótesis se realizó con la tabla de contingencia, Tabla 8.

Hn: El Nivel de Conocimientos en Negocio No se relaciona con la Experiencia en Algún Negocio de los estudiantes de ingeniería.

Ha: El Nivel de Conocimientos en Negocio si se relaciona con la Experiencia en Algún Negocio de los estudiantes de ingeniería.

Tabla 8. Conocimientos en negocio y experiencia en algún negocio

\begin{tabular}{lcccc}
\hline \multirow{2}{*}{$\begin{array}{c}\text { Eonocimientos } \\
\text { En Negocio }\end{array}$} & \multicolumn{2}{c}{ Experiencia en Algún Negocio } \\
\cline { 2 - 4 } & Producción & Servicio & Venta & Total \\
\hline Básico & 10 & 34 & 96 & 140 \\
Intermedio & 21 & 42 & 40 & 103 \\
Avanzado & 1 & 2 & 4 & 7 \\
\multicolumn{1}{r}{ Total } & 32 & 78 & 140 & 250 \\
\hline \multicolumn{1}{r}{}
\end{tabular}


Tabla 9. Correlación de Rho de Spearman

\begin{tabular}{|c|c|c|c|c|}
\hline & & & CONOCIMIENTOS & EXPERIENCIA EN \\
\hline & & & EN NEGOCIOS & ALGÚN NEGOCIO \\
\hline \multirow{7}{*}{ Rho de Spearman } & \multirow{3}{*}{$\begin{array}{l}\text { CONOCIMIENTOS } \\
\text { EN NEGOCIOS }\end{array}$} & \multirow{3}{*}{$\begin{array}{l}\text { Coeficiente de correlación } \\
\text { Sig. (bilateral) } \\
N\end{array}$} & 1,000 & $-0,284^{* *}$ \\
\hline & & & & 0,000 \\
\hline & & & 250 & 250 \\
\hline & & & & \\
\hline & FXPFRIFNCIA FN & Coeficiente de correlación & $-0,284^{* *}$ & 1,000 \\
\hline & AL GÚN NFGOCIO & Sig. (bilateral) & 0,000 & \\
\hline & & $\mathrm{N}$ & 250 & 250 \\
\hline
\end{tabular}

**. La correlación es significativa al nivel 0,01 (bilateral).

Tabla 10. Conocimientos en gestión y experiencia en algún negocio

\begin{tabular}{|c|c|c|c|c|}
\hline \multirow{2}{*}{$\begin{array}{l}\text { Conocimientos } \\
\text { En Gestión }\end{array}$} & \multicolumn{3}{|c|}{ Experiencia en Algún Negocio } & \multirow[b]{2}{*}{ Tota } \\
\hline & Producción & Servicio & Venta & \\
\hline Básico & 25 & 30 & 103 & 158 \\
\hline Intermedio & 7 & 38 & 37 & 82 \\
\hline Avanzado & 0 & 10 & 0 & 10 \\
\hline Total & 32 & 78 & 140 & 250 \\
\hline
\end{tabular}

Tabla 11. Correlación de Rho de Spearman

\begin{tabular}{|c|c|c|c|c|}
\hline & & & $\begin{array}{c}\text { CONOCIMIENTOS DE } \\
\text { GESTIÓN }\end{array}$ & $\begin{array}{l}\text { EXPERIENCIA } \\
\text { EN ALGÚN } \\
\text { NEGOCIO }\end{array}$ \\
\hline \multirow[b]{2}{*}{ Rho de Spearman } & $\begin{array}{l}\text { CONOCIMIENTOS } \\
\text { DE GESTIÓN }\end{array}$ & $\begin{array}{l}\text { Coeficiente de correlación } \\
\text { Sig. (bilateral) }\end{array}$ & $\begin{array}{l}1,000 \\
250\end{array}$ & $\begin{array}{c}-0,187^{* *} \\
0,003\end{array}$ \\
\hline & $\begin{array}{l}\text { EXPERIENCIA EN } \\
\text { ALGÚN NEGOCIO }\end{array}$ & $\begin{array}{l}\text { Coeficiente de correlación } \\
\text { Sig. (bilateral) } \\
\text { N }\end{array}$ & $\begin{array}{c}-0,187^{* *} \\
0,003 \\
250\end{array}$ & $\begin{array}{l}1,000 \\
250\end{array}$ \\
\hline
\end{tabular}

Con una significación de la muestra de 0,000, menor al 0,01 de significación probabilística, se rechaza la hipótesis nula y en su lugar se acepta la hipótesis alternativa, es decir se demuestra que el Nivel de Conocimientos en Gestión Si se relaciona con la Experiencia en Algún Negocio de los estudiantes de ingeniería.

La prueba total de hipótesis se realizó con la tabla de contingencia, Tabla 12.

Hn: El Talento Cognitivo No se relaciona con la Experiencia en Algún Negocio de los estudiantes de ingeniería.
Ha: El Talento Cognitivo Si se relaciona con la Experiencia en Algún Negocio de los estudiantes de ingeniería

Tabla 12. Talento cognitivo del estudiante $y$ experiencia en algún negocio

\begin{tabular}{lcccc}
\hline \multirow{2}{*}{$\begin{array}{c}\text { Talento } \\
\text { Cognitivo }\end{array}$} & \multicolumn{2}{c}{ Experiencia en Algún Negocio } & \\
\cline { 2 - 4 } & Producción & Servicio & Venta & Total \\
\hline Básico & 15 & 31 & 101 & 147 \\
Intermedio & 16 & 43 & 37 & 96 \\
Avanzado & 1 & 4 & 2 & 7 \\
$\quad$ Total & 32 & 78 & 140 & 250 \\
\hline
\end{tabular}


Tabla 13. Correlación de Rho de Spearman

\begin{tabular}{|c|c|c|c|c|}
\hline & & & $\begin{array}{l}\text { TALENTO } \\
\text { COGNITIVO }\end{array}$ & $\begin{array}{l}\text { EXPERIENCIA } \\
\text { EN ALGÚN } \\
\text { NEGOCIO }\end{array}$ \\
\hline \multirow{7}{*}{ Rho de Spearman } & & Coeficiente de correlación & 1,000 & $-0,285^{* *}$ \\
\hline & TALENTO & Sig. (bilateral) & & 0,000 \\
\hline & COGNITIVO & $\mathrm{N}$ & 250 & 250 \\
\hline & & & & \multirow{3}{*}{1,000} \\
\hline & \multirow{3}{*}{$\begin{array}{l}\text { EXPERIENCIA EN } \\
\text { ALGÚN NEGOCIO }\end{array}$} & Coeficiente de correlación & $-0,285^{* *}$ & \\
\hline & & Sig. (bilateral) & 0,000 & \\
\hline & & $\mathrm{N}$ & 250 & 250 \\
\hline
\end{tabular}

**. La correlación es significativa al nivel 0,01 (bilateral).

Con una significación de la muestra de 0,000, menor al 0,01 de significación probabilística, se rechaza la hipótesis nula y en su lugar se acepta la hipótesis alternativa, es decir se demuestra que el Talento Cognitivo Si se relaciona con la Experiencia en Algún Negocio de los estudiantes de ingeniería.

\section{DISCUSIÓN}

De acuerdo con los resultados de nuestra investigación, se confirma que el Talento Cognitivo se relaciona con la Experiencia en Algún Negocio de los estudiantes de la Facultad de Ingeniería Industrial, Sistemas e Informática. El Talento Cognitivo ha sido analizado a través de sus factores como; Conocimientos en Economía, Conocimientos en Negocios y Conocimientos en Gestión. Estos conocimientos en promedio tienen la categoría de básicos, lo cual indica que los estudiantes tienen limitaciones para poder emprender algún negocio de manera disciplinada y formalizada ante organismos del estado. Las mejoras de las capacidades de los estudiantes universitarios, para emprender actividades de negocios, sería una oportunidad para la población de jóvenes desempleados, quienes verían algunas alternativas nuevas de trabajos.

Sobre el tema de los desempleos juveniles, Perú21 (2015), publicó los resultados de la investigación de la empresa Aptitus.com, en ella informa que el $20 \%$ de peruanos entre 18 y 24 años tienen trabajo, esta situación se debe a las pocas oportunidades laborales que ofrece el mercado $(58 \%)$, así como a su falta de experiencia $(46 \%)$, lo que probablemente los convierta en menos atractivos para las empresas. Un pequeño grupo $(9 \%)$ reconoció que esto se debe a su falta de iniciativa propia. De este grupo de jóvenes el $64 \%$ manifestó no sentirse a gusto con su sueldo, esta realidad nos indica que los jóvenes están expuestos a ser atraídos por personas que actúan al margen de la ley con la ilusión de ganar dinero de manera rápida.

Como se entiende la situación laboral es incierta para los jóvenes universitarios, a sabiendas de que en esta edad ellos disponen de la mayor vitalidad posible, para realizar trabajos sobre todo de tipo operativos. Por esta razón se investigo el Talento Cognitivo de los estudiantes de nuestra facultad, y por el ello se recomienda realizar programas de capacitación y motivación con el fin de mejorar y fortalecer las capacidades de los estudiantes universitarios para emprender actividades de negocios y mitigar en parte el problema social del desempleo.

\section{REFERENCIAS BIBLIOGRÁFICAS}

Espitía, I. \& Barragán, E. (2004). Gerencia del Talento Humano. Colombia. Editorial ESAP.

Garavito, C. (1998). Determinantes del desempleo en Lima Metropolitana, 19701996. Economía, 21(41), 143-183.

Nel, L. (2010). Metodología de la Investigación, Estadística Aplicada en la Investigación. Lima: Empresa Editora Macro E.I.R.L.

Peru21 (2015,mayo,22). Desempleo Juvenil. Diario de Noticias, recuperado de: http://peru21.pe/economia/desempleojuvenil-solo-20-peruanos-entre-18-y-24anos-tiene-trabajo-2219335

Sánchez, H. \& Reyes, C. (1998). Metodología y Diseños en la Investigación Científica. Lima, Perú. Editorial Mantaro.

\section{Correo electrónico:}

lopez2410@yahoo.es

Revisión de pares:

Recibido: 23-05-2017

Aceptado: 28-06-2017 